\title{
Rhupus syndrome: a case report
}

\author{
Yesim Akpinar ${ }^{1 凶}$, Ülker Karagece Yalcin²
}

\begin{abstract}
Rhupus syndrome is a rare overlap syndrome that can be observed together with systemic lupus erythematosus (SLE) and rheumatoid arthritis (RA). We report the case of a young male patient diagnosed with SLE due to new skin findings and also diagnosed with RA 3 years earlier.

Keywords: rheumatoid arthritis, systemic lupus erythematosus, rhupus syndrome

Received: 21 April 2017 | Returned for modification: 31 May 2017 | Accepted: 21 June 2017
\end{abstract}

\section{Introduction}

Autoimmune diseases are known as familial chronic and systemic inflammatory disorders that can be characterized by immune system dysregulation. Rhupus syndrome is a rare disease that is clinically and immunologically observed together with rheumatoid arthritis (RA) and systemic lupus erythematosus (SLE). The prevalence of the disease is $0.09 \%$ (5). This syndrome's prevalence among systemic rheumatic diseases is estimated to be between 0.01 and $2 \%$ (12). The prognosis in rhupus syndrome depends on internal organ damage, but it is better than SLE and worse than RA. RA is responsible for Th1-mediated inflammation, whereas SLE is associated with Th2-mediated pathologies (1).

In this case study, we report the case of a 22-year-old male patient that was followed with a diagnosis of RA for the past 3 years. In our opinion, it is appropriate and beneficial to report this case study because the patient shows SLE symptoms together with RA.

\section{Case report}

A 22-year-old male patient was admitted to the hospital with complaints of redness on the face, forehead, and nose, hair loss, and joint pain. According to his medical history, pain and swelling in his knees, heel, and fingers, and especially morning stiffness in his knee, had started 3 years before he was admitted to the hospital.

His previous laboratory tests showed an erythrocyte sedimentation rate of 37 (normal o to 20), and C-reactive protein 12 (normal o to 6). The rheumatoid factor was 4 (normal o to 8), and anticyclic citrullinated peptide antibody (anti-CCP Ab) 135.9 (normal o to 20). Based on the laboratory results and typical clinical presentation, the patient had been diagnosed with RA 3 years earlier. When the patient was admitted to our clinic, he was using steroids (prednisolone $5 \mathrm{mg} /$ day).

The examination of the patient was conducted in line with the Declaration of Helsinki principles.

\section{Physical examination findings}

The patient had butterfly-style erythema on the forehead, nose, and region that contains the malar projections (Figs. 1-2). He also had erythematous papules in some areas of the inner part of the left arm.

There were alopecia plates of different diameters on various areas in the occipital scalp region (Fig. 3). There was tenderness in both knees and foot joints, and particularly in the heels, according to his joint examination. Cardiovascular, respiratory, and gastrointestinal examinations were normal.

\section{Laboratory findings}

Whole blood glucose, serum urea and creatinine levels, liver function tests (ALT, AST), and rheumatoid factor were normal. There was no fecal occult blood.

Sedimentation was 35 (normal o to 20), C-reactive protein was 15 (normal o to 6), antinuclear antibody (++fine granular appearance), anti-dsDNA antibody (+++positive), antinucleosome antibody (+++positive), anti-histone antibody (++positive), antiSmith antibody (++positive), proliferating cell nuclear antigen (PCNA) (+positive), anti SS-A/Ro (6okD) (++positive), anti-(U1)ribonucleoprotein antibody (+positive), complement 3 (C3) 44.2 $\mathrm{mg} / \mathrm{dl}$ (normal 20 to 88), and complement 4 (C4) 8.1mg/dl (normal 16 to 38 ) were normal, and HBsAg, anti-HBsAg, anti-HCV, and anti-HIV were negative. Serum iron $(\mathrm{Fe}$ ) level was $18 \mu \mathrm{g} / \mathrm{dl}$ (normal 45 to $182 \mu \mathrm{g} / \mathrm{dl}$ ). Electrocardiography and abdominal ultrasonography results were normal.

There was no lytic or sclerotic lesion in other bone structures according to a bilateral two-way hand X-ray. Joint spaces and the surfaces of both knees and feet were normal according to a twoway X-ray.

A skin biopsy was made from the erythematous plaque on the face. A skin punch biopsy showed mild acanthosis in the epidermis and in a few melanocytes in the dermis $(\mathrm{HE} \times 200)$. There were polymorphous rich inflammatory cells in the environment of the dermal veins, and a few polymorphous and core parts on the small vein wall (Fig. 4).

The patient was diagnosed with SLE and RA overlap disease according to the American College of Rheumatology (ACR) classification criteria. The patient was given $200 \mathrm{mg}$ hydroxychloroquine per day. The butterfly-like rash improved after 18 days, but he still had pain. Therefore, $20 \mathrm{mg} /$ day steroid treatment was initiated. The dose of the steroid was gradually decreased to $5 \mathrm{mg} /$ day. The joint pain lessened on the 15th day of the steroid treatment. Remission was observed after $5 \mathrm{mg} /$ day steroid and $200 \mathrm{mg}$ hydroxychloroquine treatment. He was regularly followed up at 2-month intervals. After 14 months of follow-up, he had no flareup and he was in remission. 

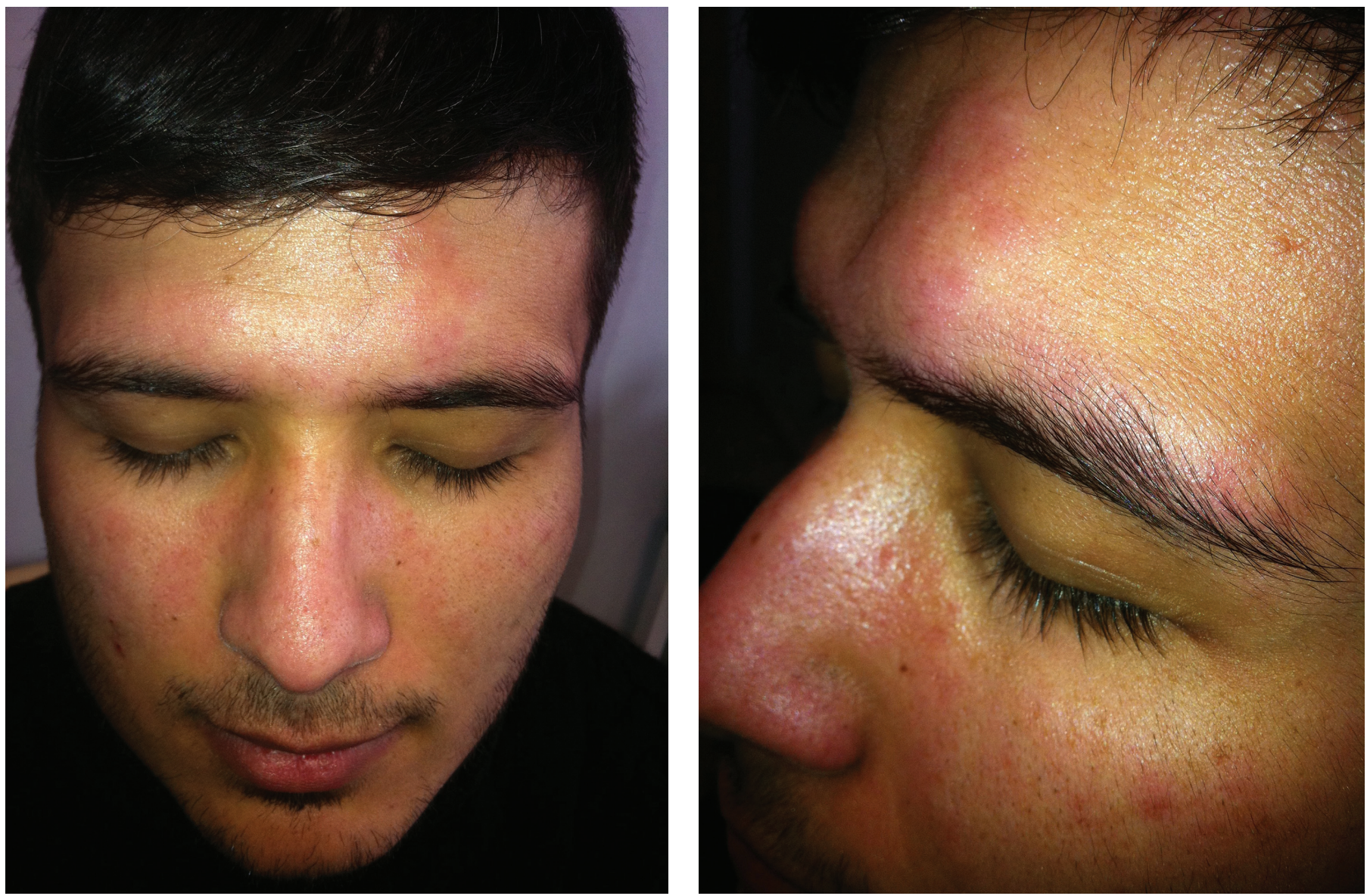

Figures 1 and 2 | Erythema on the forehead, nose, and malar region.

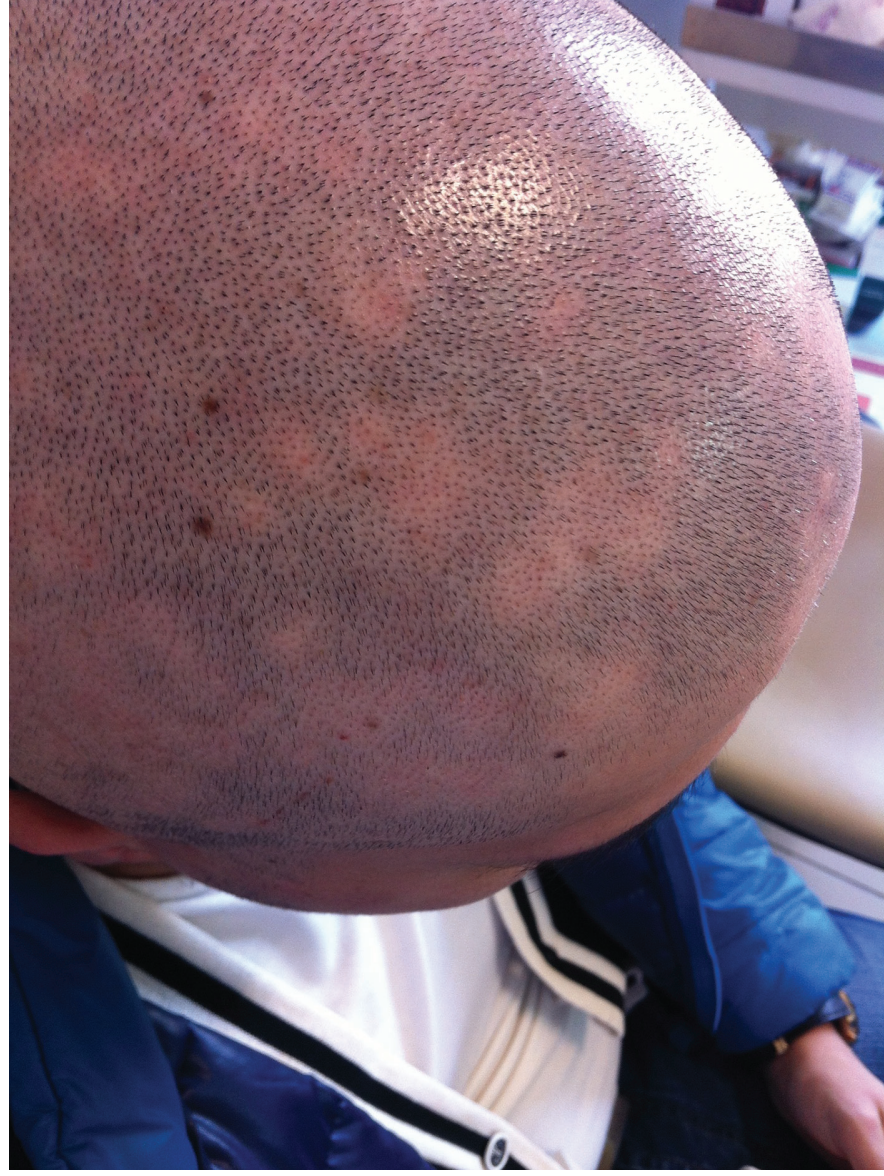

Figure 3 | Scalp alopecia areata.

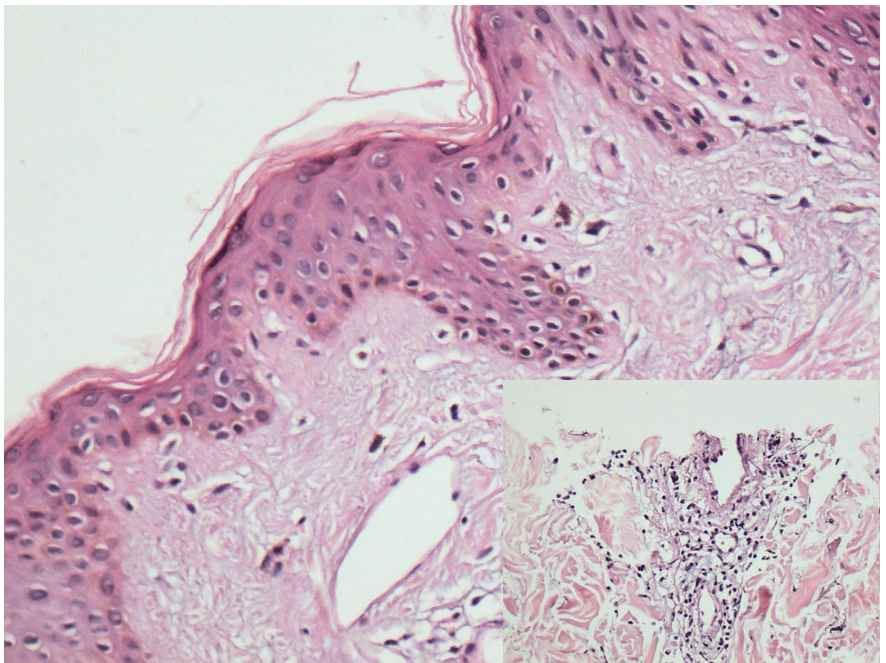

Figure 4 | Mild acanthosis in the epidermis, PMNL-rich inflammation in the dermal vein environment $(\mathrm{HE} \times 200)$.

\section{Discussion}

Overlap of RA and SLE was primarily named "rhupus syndrome" in 1974 by Schur $(2,10)$. Rhupus syndrome is generally diagnosed based on complaints such as chronic symmetric polyarthritis, butterfly-like rash, alopecia, mucosal involvement, serositis, myositis, and hematologic findings of SLE (4). The skin biopsy is not diagnostic in rhupus syndrome, but it may be necessary for differential diagnosis of other erythematous diseases such as rosacea and allergic contact dermatitis. 
Laboratory findings such as anti-CCP Ab, anti-dsDNA, or antiSmith Ab positivity are the diagnostic criteria for rhupus syndrome (6). A high titration of anti-RNP Ab (+positive) is very important in the overlap syndrome diagnosis $(3,6)$. Hypocomplementemia is not characteristic in rhupus syndrome (6). Anti-dsDNA antibodies are specific for SLE (6).

Rheumatoid factor with high sensitivity and low specificity is the diagnostic criterion for RA. On the other hand, positive antiCCP antibody has $98 \%$ specificity for diagnosis of the disease. Anti-CCP antibodies for RA are known as a more specific parameter compared to rheumatoid factor $(7,8)$.

SLE is a connective tissue disease that is characterized by the involvement of multiple systems (5). It generally starts with joint involvement, and its most frequently observed symptoms are arthritis that does not lead to deformity and myalgia. Proximal interphalangeal and metacarpophalangeal joints are primarily involved $(3,4)$.

According to ACR criteria (2012), SLE can be diagnosed when at least four out of 11 criteria are observed: malar rash, discoid rash, photosensitivity, oral ulcer, arthritis that does not cause erosion, serositis, renal involvement (proteinuria), neurological (seizures, psychosis) involvement, hematological (leukopenia and lymphopenia) involvement, immunological involvement (anti-dsDNA and anti-Sm positivity), and positive ANA $(2,6)$. Our patient had rash, alopecia, arthritis, ANA positivity, and anti-dsDNA positivity. There were four out of 11 criteria.

According to the ACR classification in 1987, RA can be diagnosed when at least four out of seven criteria are observed (joint stiffness for at least 6 weeks and morning stiffness for at least 1 hour, arthritis in at least three joints such as the knee, elbow, wrist, or ankle, arthritis in at least one hand joint (wrist, metacarpophalangeal, proximal interphalangeal joint, symmetrical arthritis, or rheumatoid nodules), serum rheumatoid factor positivity, erosion in the hand and wrist radiologically, and decalcifi- cation $(2,6)$. According to the ACR classification in 2010, which is a similar classification, our patient had two points with involvement in multiple small or large joints (knee and foot joints), three points with a high level of anti-CCp antibody positivity, one point with high-level ESR or CRP positivity, and one point with joint involvement lasting longer than 6 weeks. He had a score of $7 / 10$ based on the 2010 American College of Rheumatology/European Union League Against Rheumatism (EULAR) classification criteria for RA (11). Because the criteria for both RA and SLE were fulfilled, the diagnosis of rhupus syndrome was made.

There are similar data in the literature regarding the onset of this disease, with RA manifesting before the onset of SLE, as observed in our patient. According to previous reports; severe erosive polyarthritis and join disease occur in RA; however, non-erosive joint involvement was seen in SLE. Our patient had involvement in the knee and foot joints. Visceral organ damage in rhupus syndrome such as glomerulonephritis and serositis is less common than in SLE (12). The positivity of autoantibodies is necessary for a diagnosis. According to the literature, anti-dsDNA and anti-CCP antibodies are found with high prevalence in rhupus syndrome (12). In our patient, most of the antibodies were positive.

Low to moderate doses of corticosteroids with methotrexate are generally used in treatment with joint involvement (6). Other drugs such as mycophenolate mofetil, cyclosporin A, and tumor necrosis factor inhibitors have shown little effect on rhupus syndrome (12).

Our patient was diagnosed with SLE and RA according to ACR criteria. The diagnosis of rhupus syndrome was supported with laboratory and clinical findings. Similar to our patient, who was diagnosed with RA at a younger age, it is important to be aware of concomitant SLE development and RA patients should also be followed in terms of SLE. Therefore, we emphasize that it is crucial to examine young RA patients.

\section{References}

1. Arbuckle MR, Mc Clain MT, Rubertone MV, et al. Development of autoantibodies before the clinical onset of systemic lupus erythematosus. N Engl I Med. 2003;349:1526-33.

2. Saha K, Saha A, Mitra M, Panchadhyayee P. Bilateral pleural effusion with APLA positivity in a case of rhupus syndrome. Lung India. 2014:31:390-3.

3. Baykal C. Dermatoloji atlası. 3rd ed. Istanbul: Nobel Tıp Kitabevi. 2012.

4. Ziaee V, Moradinejad MH, Boyat R. Rhupus syndrome in children: a case series and literature review. Case Rep Rheumatol. 2013;819629.

5. Sarkar S, Saha K. Bilateral acute lupus pneumonitis in a case of rhupus syndrome. Lung India. 2012;29:280-2.

6. Verdoorn, BP, McDonald FS. 62-year-old woman with fever, dyspnea, pleuritic chest pain and weight loss. Mayo Clin Proc. 2011;86:152-5.

7. Klareskog L, Padyukov L, Rönnelid J, Alfredsson L. Genes, environment and im munity in the development of rheumatoid arthritis. Curr Opin Immunol. 2006; $18: 867-75$
8. Visser H, le Cessie S, Vos K, Breedveld FC, Hazes JM. How to diagnose rheumatoid arthritis early: a prediction model for persistent (erosive) arthritis. Arthritis Rheum. 2002;46:357-65.

9. Nakamura RM. Progress in the use of biochemical and biological markers for evaluation of rheumatoid arthritis. I Clin Lab Anal. 2000;14:305-13.

10. Schur PH. Systemic lupus erythematosus. In: Beeson PB, McDermott W, editors. Cecil-Loeb textbook of medicine. 13th ed. Philadelphia: WB Saunders; 1971. p. 821.

11. Yıldırım R, Yazıcı Y. Romatoid artritte erken tedavi. RAED. 2012;4:59-67.

12. Showman $O$, Langevitz $P$, Shoenfeld $Y$. Rhupus: unusual presentations. Clin Rheumatol. 2015;34:2041-6. 